\title{
Les plantes exotiques odorantes chez Batiouchkov et Pouchkine
}

La genèse et la fonction du motif

Igor Pilshchikov

Traducteur : Ekaterina Velmezova

\section{Q OpenEdition}

1 Journals

Édition électronique

URL : http://journals.openedition.org/edl/372

DOI : 10.4000/edl.372

ISSN : 2296-5084

Éditeur

Université de Lausanne

\section{Édition imprimée}

Date de publication : 15 septembre 2009

Pagination : 21-36

ISBN : 978-2-940331-20-8

ISSN : 0014-2026

Référence électronique

Igor Pilshchikov, "Les plantes exotiques odorantes chez Batiouchkov et Pouchkine », Études de lettres [En ligne], 2-3 | 2009, mis en ligne le 15 septembre 2012, consulté le 19 décembre 2020. URL : http:// journals.openedition.org/edl/372; DOI : https://doi.org/10.4000/edl.372 


\section{LES PLANTES EXOTIQUES ODORANTES CHEZ BATIOUCHKOV ET POUCHKINE: LA GENĖSE ET LA FONCTION DU MOTIF}

Le présent article suit le parcours de plusieurs noms de plantes qui fonctionnent comme autant d'indices de l'exotique - antique, biblique ou nordique - dans la poésie russe des premières décennies du XIX ${ }^{\mathrm{e}}$ siècle. Ainsi est observée la richesse combinatoire d'un motif qui peut donner lieu à un cliché stylistique autant qu'à un usage lyrique ou ironique et auto-réflexif.

Propre au classicisme tardif et au premier romantisme, l'intérêt pour les cultures chronologiquement et géographiquement lointaines contribue à l'établissement, dans la poésie russe du début du XIX ${ }^{\mathrm{e}}$ siècle, de parallèles associant les Antiquités «septentrionales» (celtes, scandinaves, finlandaises, voire slaves), bibliques et gréco-romaines (classiques).

Nous avons déjà montré par ailleurs ${ }^{1}$ que les éléments clefs du cantique funèbre de David déplorant la mort de Saül et de Jonathan (II Sam. 1) sont présents dans les trois principaux textes élégiaques de l'ossianisme russe, à savoir le Chant du barde sur le tombeau des Slaves vainqueurs (Pesn' Barda nad grobom Slavjan-pobeditelej, 1806) de Vassili Joukovski, l'élégie historique de Konstantin Batiouchkov Sur les ruines d'un château en Suède (Na razvalinax zamka v Švecii, 1815) et l'élégie d'Evgueni Baratynski Finlande (Finljandija, 1820). Ces textes établissent des parallèles entre les Antiquités scandinave et biblique, qui sont typiques de la poésie du premier romantisme.

I. Cf. I. A. Pil’ščikov, «Iz nabljudenij nad genezisom i poètikoj èlegij Baratynskogo », p. 60-61. 
Les Antiquités biblique et classique se retrouvent également liées chez les poètes russes de l'«Age d'or ", d'où une autre ligne de l'évolution stylistique de la poésie russe (essentiellement élégiaque) : la présence, dans le texte élégiaque russe, de sujets érotiques antiques où sont réunies les phraséologies de la Bible et de l'élégie de la Rome antique. Dans cet article, nous analyserons ce phénomène à partir du lexique désignant les plantes exotiques odorantes dans la traduction russe d'une des élégies de Tibulle (effectuée par Batiouchkov) et dans l'imitation pouchkinienne du Cantique des Cantiques.

Notre brève description des deux orientations de l'élégie russe fait apparaître par deux fois le nom de Konstantin Batiouchkov. Il est, en Russie, l'un des pionniers de l'ossianisme élégiaque, ainsi que de l'élégie d'amour (orientée vers les œuvres latines classiques). Ce n'est pas un hasard. Le jeune Batiouchkov passe plusieurs années chez son oncle Mikhaïl Mouraviev, l'un des grands poètes du sentimentalisme russe, créateur de la "poésie légère». Fervent admirateur de l'Antiquité, Mouraviev enseigne à son neveu la langue latine et la littérature de la Rome antique. C'est probablement dans la maison de son oncle que Batiouchkov se lie d'amitié avec Alexis Olenine, haut fonctionnaire et connaisseur des $\operatorname{arts}^{2}$. Le cercle d'Olenine, centre esthétique du néoclassicisme russe ou (d'après la définition de Boris Tomachevski) du «style Empire» russe, est connu pour son goût des Antiquités nordiques ${ }^{3}$. Nikolaï Gneditch, futur traducteur de l'Iliade en russe, est un autre habitué du cercle et devient l'ami le plus proche de Batiouchkov.

En 1800-1810, Batiouchkov et Joukovski font de l'élégie le genre majeur de la poésie russe: Joukovski transporte en terrain russe la poétique de l'élégie méditative anglaise, et Batiouchkov de l'élégie érotique latine. Tibulle ne tarde pas à représenter pour ce dernier le poète romain par excellence.

La traduction de Tibulle par Batiouchkov aura une grande influence sur la poétique et la stylistique de l'élégie russe érotique et érotiquerustique. Dans les années 1810, Tibulle devient en Russie l'élégiaque le plus populaire de l'époque d'Auguste, ses élégies en vers alexandrins

2. Plus tard, Batiouchkov dédicacera son élégie Hésiode et Homère, rivaux [Geziod i Omir, soperniki] à Olenine: «Pour A. N. O., amateur de l'Antiquité».

3. B. Tomaševskij, «K. N . Batjuškov», p. XXIII-XXVII. 
sont synonymes de l'élégie antique en tant que telle ${ }^{4}$. Il faut attendre les années 1820 pour qu'Ovide lui ravisse la première place au Parnasse élégiaque russe (ce qui mérite d'être souligné, car, en France par exemple, la réception historique des élégiaques romains se passe très différemment ${ }^{5}$ ).

Une autre particularité intéressante de la poétique et de l'esthétique de Batiouchkov (qui le rapproche également de Joukovski) tient à la passion du poète pour l'exotisme. Batiouchkov oppose, à plusieurs reprises, le "familier» (au sens de «sien", "qui appartient en propre») et l'"étranger", et, ce faisant, révèle sa préférence pour l'«étranger». (Nous avons analysé en détail l'interaction du "familier» et de "l'étranger» dans l'héritage épistolaire du poète ${ }^{6}$ ). Il nous suffira ici de rappeler qu'un carnet de Batiouchkov, daté de 1817, porte l'inscription: L'Etranger, mon trésor! (Čužoe: moe sokrovišče!).

Le degré d'altérité de l'étranger peut varier. L'étude des cultures russe et européenne du XVIII et du début du XIXe siècles se heurte d'emblée au problème des textes et littératures qui ont servi d'intermédiaires. Le plus exotique est toujours introduit par le biais d'un intermédiaire moins exotique: ainsi la littérature allemande est-elle assimilée, en Russie, avec l'aide de la littérature française; l'anglaise à travers la française et l'allemande; la grecque à travers l'allemande; la latine à travers la française. Si le français est "étranger" du point de vue de la langue russe, il ne l'est pas par rapport au latin: à sa sœur soucieuse de l'éducation de son fils, Batiouchkov écrit que la langue latine est un élément indispensable de l'éducation à la maison, mais qu'il n'est pas nécessaire d'apprendre le français: cela se fera tout seul, "dans les conversations de tous les jours» (v razgovore $)^{7}$.

De la même façon, pour assimiler un nouvel exotique (nordique et antique), Batiouchkov (et, dans ce cas précis, d'autres poètes russes de

4. Cf. I. A. Pil’ščikov, «O roli versij-posrednikov pri sozdanii perevodnogo teksta: (Dmitriev - Lagarp - Skaliger - Tibull)»; id., " "Ja vozvraščusja k vam, polja moix otcov..." : Baratynskij i Tibull», p. 30-33, etc.

5. Cf. p. ex. H. Potez, L'Elégie en France avant le Romantisme (de Parny à Lamartine), 1778-1820; K. Jungman, Studien zur französische Elegie des 18. Jahrhunderts mit besonderer Berücksichtung der Tibull-Rezeption.

6. Cf. I. A. Pil'ščikov, Batjuškov i literatura Italii: Filologičeskie razyskanija, p. 116117 et suiv.

7. K. N. Batjuškov, Sočinenija, p. 381-383. 
l'«Age d'or») s'adresse à un exotique plus connu: l'exotique biblique. Ce recours aux biblismes est favorisé par la situation particulière de la langue de la Bible slave. D'une part, pour contrer l'esthétique linguistique des "archaïsants» et "néo-archaïsants», les adeptes de Karamzine de l'ancienne génération, ainsi que les "néo-karamzinistes" (Batiouchkov, notamment) opposent le slavon d'Eglise au russe. D'autre part, de nombreux slavonismes sont parfaitement assimilés par la langue russe dite littéraire; c'est pourquoi Joukovski, Batiouchkov et, plus encore, Pouchkine utilisent ces éléments slavons marqués au coin d'une altérité (inakost') historique et culturelle, comme s'ils faisaient partie de "leur» langue ${ }^{8}$.

Venons-en, à présent, au sujet de cet article proprement dit: les plantes exotiques odorantes dans la poésie de Batiouchkov et de Pouchkine.

Dans sa traduction de la troisième élégie du premier livre de Tibulle, Batiouchkov décrit ainsi L'Elysée des amants (Elizij ljubovnikov, la traduction est faite en 1810-1811 et publiée en $1815^{9}$ ):

Là-bas c'est l'éternel printemps entre les bocages et les champs,

Le nard odorant et le cinnamome sont en fleur

Et l'air est embaumé du parfum de la rose ${ }^{10}$.

Or, chez Tibulle (Elégies, I, 3, 61-62), il est question de cinnamome (ou de canelle-casia) et de roses (rosae):

[...] fert casiam non culta seges, totosque per agros

floret odoratis terra benigna rosis $[\ldots]^{11}$.

8. La question des liens entre les exotismes et les archaïsmes dans la poésie de Pouchkine fut posée par Viktor Vinogradov dans ses travaux d'avant-guerre (cf. V. V. Vinogradov, Jazyk Puškina: Puškin i istorija russkogo literaturnogo jazyka, p. 121 et suiv.).

9. Cf. I. A. Pil’ščikov, «Simvolika Elizija v poèzii Batjuškova» (certaines thèses discutées dans le présent article ont été exposées pour la première fois dans ce travail). Sur la datation de la traduction de Tibulle, I, 3 par Batiouchkov, cf. ibid., p. 89, 113, note 5; I. A. Pil’ščikov, "Tibullovy èlegii v perevode Batjuškova: Materialy dlja akademičeskogo kommentarija», p. 310-311.

IO. "Tam večnaja vesna mež roščej i polej, // Cvetet dušistyj nard i kinnamona lozy, // I vozduh napoen blagouhan'em rozy [...]", (K. N. Batjuškov, "Tibullova Elegija. [Kniga 1. Elegija 3.]", p. 208).

II. Tibulle et les auteurs $d u$ corps tibullianum, texte établi et traduit par M. Ponchont, p. 26: «[...] le cinnamome y pousse sans culture, et dans toute la campagne la terre 
Voici comment ces vers apparaissent sous la plume d'un autre traducteur de Tibulle, Pavel Golenichtchev-Koutouzov, prédécesseur et adversaire de Batiouchkov, puisque se rattachant au camp des «archaïsants»:

Là où le printemps éternel brille parmi les fleurs,

Là où le cinnamome [la cannelle] embaumé(e) pousse,

Là où la rose fraîche embaume ${ }^{12}$.

Batiouchkov traduit casia par cinnamome (kinnamon en slavon $<\mathrm{du}$ grec et hébreu, cf. en latin cinnamomum, cinnamon, cinnamum) et mentionne une autre plante: "le nard odorant» (dušistyj nard). Chez Tibulle, l'épithète odoratus, "odorant", se rapporte uniquement aux roses (odoratis rosis, "roses odorantes»). La traduction de Batiouchkov présente donc, dans deux vers voisins, deux termes russes sémantiquement identiques (dušistyj, "embaumé», et blagouxan'e, "arome, parfum»), alors qu'il en est un seul dans l'original latin. C'est probablement pour cette raison que l'épithète dušistyj fut supprimé dans la version définitive de cette traduction, parue dans les Essais en vers et en prose (Opyty $v$ Stixax $i$ Proze) de Batiouchkov, en 1817:

Là où fleurissent le nard et le cinnamome,

Où l'air est embaumé du parfum de la rose ${ }^{13}$.

Dans l'œuvre de Tibulle (Elégies, II, 2, 7 ; III, 6, 63), le mot nardus / nardum est utilisé deux fois, toujours au sens d'" huile de nard ${ }^{14}$ (et non de "plante»), sans aucun lien avec l'Elysée ou le cinnamome. Dès lors, se pose la question: sur quoi le poète russe se fonde-t-il

complaisante est fleurie de roses embaumées». Cf. la traduction du comte de Mirabeau : "[...] les campagnes odorantes portent sans culture la canelle et les roses parfumées» (Elégies de Tibulle, t. II, p. 115).

I2. "[...] Gde večnaja vesna blistaet sred' cvetov; // Gde blagovonnaja korica vozrastaet, // Gde roza svežaja vsegda blagouhaet [...]»(P. Golenǐ̌čev-Kutuzov, Stihotvorenija, p. 298).

I3. "[...] Gde rascvetaet nard i kinnamona lozy // I vozdux napoen blagouhan'em rozy [...]» (K. N. Batjuškov, Opyty v Stihax i Proze, p. 24).

I4. Cf. «[...] des gouttelettes d'un nard pur découlent de ses tempes» (Tibulle et les auteurs du corps tibullianum, p. 90); "[...] j'aurais dû arroser mes tempes du nard de Syrie» (ibid., p. 154) (NdT). 
pour modifier ces vers classiques? Le recours à d'autres textes de poètes latins ne nous sera d'aucune aide, nous le verrons, pour répondre à cette interrogation. Virgile (Enéide, VI, 658), certes, aurait pu nous fournir quelques précieuses informations, puisque, à l'en croire, aux ChampsElysées les bienheureux festoient ("mangent» et "chantent») "dessous un bois de verts lauriers" (inter odoratum lauri nemus) ${ }^{15}$. Batiouchkov se remémore manifestement ce passage de l'Enéide quand il écrit sa Vision sur les rives du Léthé (Videnie na bregax Lety, 1809), dont l'action se déroule "dans l'Elysée sacré, // à l'ombre du bocage de lauriers» (v Elizii sviaščennom, // Lavrovym lesom osenennym). Les lauriers mentionnés par Virgile se retrouvent, avec l'arbre de Vénus (le myrte), dans la traduction du passage étudié de la Troisième élégie de Tibulle, effectuée par Alexis Merzliakov (cette traduction date du milieu des années 1810, mais elle n'est publiée qu'en 1826):

[...] Le laurier et le myrte, s'entrelaçant, s'adonnent à la joie;

Dans les champs, la douceur des roses immortelles embaume ${ }^{16}$.

Mais dans la description des Champs élyséens de Virgile, il n'y a pas trace de nard ou de cinnamome. Chez Horace, qui parle des champs des bienheureux dans la XVI épode, on trouve les plantes suivantes: vinea, "la vigne", olivae, "l'olivier", ficus, "le figuier" [la figue], ilex, «l'yeuse» (44-47) ${ }^{17}$. Le nard, la cannelle et le cinnamome sont mentionnés dans le XVe livre des Métamorphoses, dans la description du phénix qui «se renouvelle et se recrée lui-même» tous les cinq siècles (Ovide, Les Métamorphoses, XV, 395-400) ${ }^{18}$; mais cela n'a rien à voir avec l'Elysée des amants de Tibulle.

C’est dans la poésie hébraïque, et non latine, que nous trouverons les contextes recherchés: pour Batiouchkov et ses contemporains, le rapport

I5. Virgile, Euvres complètes, traduites du latin par J.-P. Chausserie-Laprée.

I6. "[...] Lavr s mirtom sopletjas', lelejut v kuščah radost'; // V poljah - bessmertnyh roz blagouhaet sladost' " (A. Merzljakov, Podražanija i perevody iz Grečeskih i Latinskih stixotvorcev, p. 276).

I7. " [...] où, toujours, la vigne fleurit sans qu'on l'émonde; où bourgeonne le rameau d'un olivier qui jamais ne trompe; où la figue brune décore un arbre qui est le sien; où le miel coule du creux de l'yeuse». Horace, Odes et épodes, p. 226 (NdT).

I8. "[...] il [le phénix. - E. V.] amasse de la cannelle, des épis du nard odorant, des morceaux de cinname [...]», Ovide, Les Métamorphoses, p. 134 (NdT). 
entre le nard et l'ancien Orient (donc l'Orient biblique) est évident. Ainsi l'auteur d'une charade publiée dans la revue Le Messager de l'Europe (Vestnik Evropy) propose-t-il, soufflant en même temps la réponse à ceux de ses lecteurs qui ont l'esprit plus lent, de deviner comment est désigné, dans la Bible, un célèbre parfum: "le nard" ${ }^{19}$. Nous sommes donc parfaitement fondés à supposer que l'association "nard - cinnamome" (précisément le "cinnamome», et non la "cannelle») surgit pour Batiouchkov sous l'influence du Cantique des Cantiques, à la traduction duquel il travaille dans la deuxième moitié de 1810, autrement dit durant les mois où il traduit l'élégie de Tibulle. Cette traduction n'a pas été conservée, mais elle est mentionnée dans la correspondance de Batiouchkov avec Gneditch et Viazemski. A la fin du Chapitre IV du Cantique des Cantiques est décrit "un jardin fermé» où, parmi d'autres plantes odorantes, sont mentionnés le nard (BHS נרדז nērd, LXX

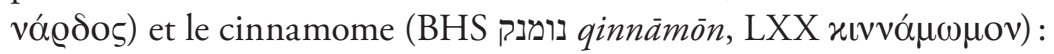

[...] des troènes et du nard; du nard et du safran, du roseau aromatique et du cinnamome, avec tous les arbres (qui donnent) de l'encens; de la myrrhe et de l'aloès, avec tous les meilleurs aromates [... ${ }^{20}$.

Dans l'imitation pouchkinienne de ces lignes ( Dans le verger de ma sœur», "Vertograd moej sestry...») le nard et le cinnamome sont présents dans le même vers:

Que le nard est odorant,

L'aloès et la cannelle:

Dès que vient souffler l'Autan,

Les parfums naissent, ruissellent ${ }^{21}$.

Soulignons que, dans la poésie de Pouchkine et de Batiouchkov, l'hellénisme nard et l'hébraïsme kinnamon ("cinnamome») ne sont mentionnés qu'une fois. Ce sont des mots rares, conditionnés par le contexte historique et culturel.

19. Anonyme, "Charady", in Vestnik Evropy, 1820, partie CXI, 10, p. 155; ibid., 11, p. 231.

20. Cantique des Cantiques $4: 13-14$.

2I. Traduction française d'A. Markowicz dans A. Pouchkine, Euvres complètes, p. 91. «Nard, aloj i kinnamon // Blagovoniem bogaty: // Liš poveet akvilon, // I zakapljut aromaty» (A. Puškin, Stihotvorenija, p. 57). 
Il est à noter que ce contexte ne contredit pas le contexte latin. Il n'y a pas de nard (au sens de plante) dans l'œuvre de Tibulle, mais d'autres poètes romains (par exemple, Horace et Ovide) parlent eux aussi du nard comme d'un parfum oriental. En même temps, chez Pouchkine, on trouve un mélange typique de slave et de latin. A l'«aquilon" (akvilon) de Pouchkine ${ }^{22}$, correspond, dans ce que l'on appelle couramment la Bible d'Elisabeth ${ }^{23}$, "le vent du nord" (sever), et dans la Vulgate - l'aquilo: "Eveille-toi, (vent du) nord! Viens, (vent du) sud! Souffle sur mon jardin, et que ses aromates s'en exhalent!»; «surge aquilo et veni auster perfla hortum meum et fluant aromata illius» (Cantique des Cantiques 4 : 16). Au demeurant, il est probable que Pouchkine emprunte le terme aquilon à la traduction française de la Vulgate, plutôt qu'au texte lui-même (c'est en tout cas le point de vue de Mikhaïl Mourianov) ${ }^{24}$.

Pour Batiouchkov, le Cantique des Cantiques est non seulement un texte religieux, mais aussi une ouvre caractéristique de la poésie d'amour antique, ce qui le rend comparable à l'élégie érotique de Tibulle. En outre, dans une lettre à Viazemski du 26 juillet 1810, le poète souligne tout spécialement le caractère érotique du Cantique des Cantiques:

Mais, trêve de plaisanterie, je suis maintenant occupé. A quoi? Devine! Je traduis le Cantique des Cantiques en vers. Quand j'en aurai terminé, je te l'enverrai, à toi, mon Aristarque, ma Vierge à déflorer (na rastlenie) [...] je - ou plutôt ma muse - vagabonde maintenant sur les hauteurs de Sion, sur les rives du Jourdain, sur les collines fraîches de l'Engadi, donc, comme je te l'ai dit, je suis si occupé par mon travail sur le Cantique des Cantiques que je vois des Juifs partout, même en rêve, et hier encore, dans mes pensées, j’ai eu (uestestvil) la Vierge de Judée ${ }^{25}$.

22. L'«Autan», dans la traduction française, cf. la note précédente (NdT).

23. Bible éditée sous le règne d'Elisabeth Petrovna (1709-1762), impératrice de Russie en 1741-1762 (NdT).

24. M. F. Mur'janov, «K strukture obraza puškinskoj Tat’jany», p. 216-217; cf. G. Safran, "Love Song Between the Sacred and the Vernacular: Pushkin's "Podrazhaniia" in the Context of Bible Translation", p. 170.

25. K. N. Batjuškov, Sočinenija, p. 141 ; cf. M. I. Šapir, «Iz istorii russkogo "balladnogo stixa": Perom vladeet kak eldoj", p. 68. 
Toutefois, ni Gneditch ni Viazemski n'apprécient cette traduction du Cantique des Cantiques. Viazemski, irrité, écrit à Batiouchkov:

Ton Cantique des cantiques aura raison de moi! De grâce, à quoi cela ressemble-t-il? Une fille désirant attirer son amant chez elle, lui dit qu'elle lui a préparé du safran? Je suis inculte, je l'avoue, je ne connais ni les habitudes ni les mœurs des Anciens et ne puis donc juger [...] mais je crois qu'une fille de notre siècle ne séduira personne ni ne se laissera séduire par du safran ou de la rhubarbe (reven') ${ }^{26}$.

Hormis le $14^{\mathrm{e}}$ verset du Chapitre IV du Cantique des Cantiques, le

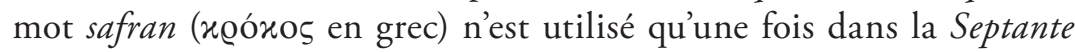
et dans la Bible d'Elisabeth: dans les Proverbes de Salomon, lorsqu'une femme tente de séduire un jeune homme privé de raison: «[...] j’ai parfumé ma couche de myrrhe, d'aloès et de cinnamome. Viens, enivronsnous de volupté jusqu'au matin, livrons-nous aux plaisirs de l'amour" (Proverbes 7 : 17-18). Selon toute vraisemblance, Batiouchkov s'en est inspiré dans sa traduction du Cantique des Cantiques.

Presque un demi-siècle plus tard, cet épisode des Proverbes de Salomon entre, malgré tout, dans la poésie russe. On le retrouve dans le poème de Lev Meï Imitation des orientaux (Podražanie vostočnym, 1856) (et non «Imitation des Anciens»!), où une jeune femme séduit un jeune sot (glupec molodoj):

Elle le verra, elle l'entendra de loin,

Et elle sortira et se mettra à le caresser,

Et elle se mettra à murmurer

Ses paroles perfides d'un air effronté: «[...] Je t’ai cherché, cherché

Et attendu depuis le soir:

J'ai couvert le lit d'un rideau et étendu

Les doubles tapis d'Egypte,

J'ai couvert la couche de safran,

Et le plancher de cannelle [de cinnamome] -

Viens et, dans une joie désirée

Nous nous allongerons près de la table.

Mon mari est parti pour un voyage lointain

Et il a emporté beaucoup d'argent,

26. P. A. Vjazemskij, «Pis’ma k K. N. Batjuškovu», p. 121-122. 
Ainsi que son œil jaloux:

Reste chez moi jusqu'au matin...»

Elle l'a séduit par une conversation pécheresse,

Elle l'a attiré dans les filets de sa bouche -

Et à la suite de cette femme, en hâte,

La folle victime s'en est allée ${ }^{27}$.

Chez Meï, on trouve également une imitation de la description du «jardin fermé» du Cantique des Cantiques (1859):

Voilà pourquoi tu as si tôt

Enflammé en nous les rêves passionnés,

Tu nous as séduit avec ton regard

Et tu es devenue comme notre jardin bien-aimé,

Là où se trouve une source fermée,

Là où tout est en fleur: le nard et le safran,

Le cyprès et le cinnamome,

Là où sont plus vertes qu'au-dessus du Liban

Toutes les herbes...

Vite, vite,

souffle avec la fraîcheur matinale

Dans notre jardin, du nord et du sud,

Ah vent! Je t'attends comme un ami...28

27. "Uvidit - uslyšit daleče, // I vyjdet, i stanet laskat', // I stanet kovarnye reči // S besstydnym licom lepetat': // [...] Tebja ja iskala, iskala - // Ždala ot večernej pory: // Zavesila odr i postlala // Egipta dvojnye kovry, // Posypala lože šafranom, // Koricej posypala pol - // Vojdi i v vesel'e želannom // Vozljažem za trapeznyj stol. // Moj muž otlučilsja dalëko // I mnogo unes serebra - // Unes i revnivoe oko - // Probud' u menja do utra... // Prel'stila besedoju grešnoj, // Tenëtami ust privlekla, - // I vsled za ženoju pospešno // Bezumnaja žertva pošla».

28. "Vot otčego tak rano ty // Zažgla v nas strastnye mečty, // Tak rano nas prel'stila vzgljadom // I vyrosla ljubimym sadom, // Gde ključ u nas zapečatlën, // Gde vsë cvetet: i nard s šafranom, // I kiparis, i kinnamon, // Gde zelenej, čem nad Livanom, // Vsja letorosl'... // Skorej, skorej // Prohladoj utrennej povej // V naš sad, i s severa i s juga, // O veter!... Ždu tebja, kak druga...». 
Il semble qu'il n'y ait pas, dans la poésie russe, d'autre contexte où soient présents, en même temps, "le nard, le safran et le cinnamome», ou ne fût-ce que «le nard et le cinnamome». Cela tendrait à confirmer, une nouvelle fois, l'exclusivité de l'inspiration biblique de ces images ${ }^{29}$.

On trouve toutefois, dans la poésie mondiale, un micro-contexte où le nard voisine avec le cinnamome: dans le Livre des Baisers (Liber basiorum), IV (1534-1535) du poète néo-latin Jean Second (Joannes Secundus):
Non dat basia, dat Neaera nectar, dat rores animae suaveolentes, dat nardumque, thymumque, cinnamumque $[\ldots]^{30}$.

Montaigne, l'un des écrivains préférés de Batiouchkov, place les Baisers de Jean Second au niveau des grands livres de Boccace et de Rabelais (Essais, livre II, chapitre 10). Batiouchkov, lui, lit Le Livre des Baisers dans l'original ou en parallèle avec la traduction française, et traduit librement le II" Baiser sous le titre «L'Elysée» («Elizij») ${ }^{31}$.

On notera que les quatre plantes odorantes mentionnées dans le Cantique des Cantiques (le nard, le safran, le roseau aromatique [xó $\lambda \alpha \mu \mathrm{s}]$ et le cinnamome) comptent parmi les plantes du paradis dans le pseudo-épigraphe judéo-chrétien Vie grecque d'Adam et Eve, connu

29. Nous ne pouvons exclure que l'on trouve chez Fiodor Sologoub, qui aimait beaucoup jouer avec les exotismes, d'un côté, et avec les paronymes, de l'autre, un reflet de cet ensemble de motifs: Šafran i kardamon, i tomnuju vanil'/l Vmešal ja v omeg moj $i v$ son bagrjanyh makov ("Le safran et le cardamome, et la vanille langoureuse // J'ai mêlé avec de la ciguë et avec le rêve des pavots purpurins») ("Sur les collines de l'aurore, une vraie histoire mystérieuse...» ["Na holmah zarevyh tainstvennuju byl’...»], 1907; le cycle «Le festin purpurin de l'aurore» [«Bagrjanyj pir zari»]); plus loin dans cette poésie surgit le sujet de la tentation sexuelle. Sur la poétique des exotismes chez Sologoub, cf. M. Šapir, «Simvoličeskaja zaum’ Fedora Sologuba: meždu lož ju i fantaziej».

30. Cf. dans la traduction française de M. Rat: "Elle ne donne pas des baisers, Néère, elle donne du nectar, elle donne la rosée suavement odorante de son haleine, elle donne du nard, du thym, du cinname [...]» (J. Second, Les Baisers et l'épithalame suivis des odes et des élégies, p. 7). Les traductions en russe ont été réalisées par Sergej Solov'ev ("III livre des poésies», 1909-1912; cf. S. Solov’ev, Sobranie stihotvorenij, p. 365) et par Sergej Šervinskij (Erazm Rotterdamskij, Stihotvorenija; Ioann Sekund, Pocelui. Edition préparée par M. L. Gasparov, S. V. Šervinskij, Ju. F. Šul'c, p. 219).

3I. Cf. I. A. Pil’ščikov, «Simvolika Elizija v poèzii Batjuškova», p. 96-111. 
aussi sous le titre d'Apocalypse de Moïse (Ap. Mos. 29 : 6; Vita 43 : 3). Cependant, rien ne permet de penser que Batiouchkov connaissait cette œuvre curieuse, d'autant que dans la version slave du livre d'Adam (à la différence des versions grecque et latine) il n'y a pas d'énumération de plantes odorantes. Il ne fait aucun doute, en revanche, que notre poète lie la chaîne sémantique "parfums orientaux" (... nard - safran aloès - cinnamome - ...) à un autre "paradis", exotique et érotique. Le 19 août 1809, Batiouchkov demande à Gneditch de lui envoyer du tabac «turc, du bon, le meilleur qui soit, tel qu'on n'eût pas honte d'en fumer au paradis de Mahomet, en compagnie des Houris, avec des Parfums d'Arabie, avec de l'aloès, du safran, des anémones, du jus d'ananas... Mais, tu me comprends!...» ${ }^{32}$

D'où viennent, en ce début du XIX ${ }^{\mathrm{e}}$ siècle, de telles idées sur le paradis musulman, nous l'ignorons encore. Elles réapparaissent, d'ailleurs, dans le «Voyage d'Oneguine»:

Puis, vivifié par l'eau cinglante,

Je fume une pipe brûlante

Et, musulman au paradis,

Bois un café qui s'épaissit ${ }^{33}$.

Une de leurs sources pourrait être le roman pseudo-oriental français du XVIII ${ }^{e}$ siècle, ainsi que ses traces dans la poésie française, tant narrative que lyrique. Ainsi revenons-nous au thème de la médiation culturelle, de l'exotique plus ou moins "étranger». Mais cela constitue le sujet d'une autre recherche.

Igor Pilshchikov

Moscou

Traduction Ekaterina Velmezova

32. K. N. Batjuškov, Sočinenija, p. 99.

33. Traduction française d'A. Markowicz dans A. Pouchkine, Eugène Onéguine, p. 239. «Potom za trubkoj raskalennoj // Volnoj solenoj oživlennoj, // Kak Musul'man v svoem raju, // S vostočnoj guščej kofe p'ju». 


\section{BIBLIOGRAPHIE}

Anonyme, "Charady", in Vestnik Evropy, 1820, partie CXI, 10, p. 155-156; «[Otvety]», ibid., 11, p. 231.

Batjuš́ov, K. N., «Tibullova Èlegija. (Kniga 1. Èlegija 3.)», in Panteon Russkoj Poèzii, Izdavaemyj P. Nikol'skim, partie IV, livraison 8, Sankt-Peterburg, Akademija nauk, 1815, p. 204-211.

—, Opyty v Stihah i Proze, St. Petersburg, N. Greč, 1817.

-, Sočinenija, tome 2, Iz zapisnyh knižek; Pis'ma, Moskva, Hudožestvennaja literatura, 1989.

Gasparov, M., Šervinskij, S., Šul'c, Ju. (éd.), tr. Erazm Rotterdamskij, Stihotvorenija. Ioann Sekund, Pocelui, Moskva, Nauka, 1983.

GoleniščEv-Kutuzov, P., Stihotvorenija, partie IV, Soderžaščaja sočinenija, perevody i podražanija, Moskva, Universitetskaja tipografija, 1810.

Horace, Odes et épodes, Tome I, établi et tr. par F. Villeneuve, Paris, Les Belles lettres, 1967.

Jungman, K., Studien zur französischen Elegie des 18. Jahrhunderts mit besonderer Berücksichtung der Tibull-Rezeption, Hamburg, Romanisches Seminar, 1976.

MEJ, L. A. Izbrannye proizvedenija, Leningrad, Sovetskij pisatel', 1972.

Merzljakov, A., Podražanija i perevody iz Grečeskih i Latinskih stihotvorcev, partie II, Moskva, Universitetskaja tipografija, 1826.

Mirabeau [H.-G. Riqueti, Comte de], tr., Elégies de Tibulle, avec des notes et recherches de Mythologie, d'Histoire et de Philosophie. Suivies des Baisers de Jean Second, tome II, traduction nouvelle, adressée du Donjeon de Vincennes, par Mirabeau l'aîné, à Sophie Ruffey, Tours-Paris, L'an III de l'Ere Républicaine [1794].

Mur'Janov, M. F., "K strukture obraza puškinskoj Tat'jany», in Problemy strukturnoj lingvistiki 1980, Moskva, Nauka, 1982, p. 213-222. 
Ovide, Les Métamorphoses, t. 3 (XI-XV), texte établi et tr. par G. Lafaye, Paris, Les Belles Lettres, 1966.

PiL’ščı́ov, I. A., " Ja vozvraščusja k vam, polja moih otcov...”: Baratynskij i Tibull", Izvestija RAN. Serija literatury i jazyka, 53, 2 (1994), p. 29-47.

—, «O roli versij-posrednikov pri sozdanii perevodnogo teksta: (Dmitriev - Lagarp - Skaliger - Tibull) ", Philologica, 2, 3/4 (1995), p. 87-111.

—, "Iz nabljudenij nad genezisom i poètikoj èlegij Baratynskogo", Izvestija RAN. Serija literatury i jazyka, 66, 3 (1997), p. 57-64.

—, Batjuškov i literatura Italii: Filologičeskie razyskanija, Moskva, Jazyki slavianskoj kul'tury, 2003.

—, "Simvolika Elizija v poèzii Batjuškova», in Antropologija kul'tury, vol. 2, Moskva, Verdana, 2004, p. 86-123.

—, "Tibullovy èlegii v perevode Batjuškova: Materialy dlja akademičeskogo kommentarija", in Tekst i kommentarij: Kruglyj stol k 75-letiju Vjačeslava Vsevolodoviča Ivanova, Moskva, Nauka, 2006, p. 267-336.

Ponchont, Max (éd.), Tibulle et les auteurs du corps tibullianum, Paris, Les Belles lettres, 1967.

Potez, Henri, L'Elégie en France avant le Romantisme (de Parny à Lamartine), 1778-1820, Paris, Calmann-Lévy, 1898.

Pouchine, A., Euvres complètes, t. 2. Euvres poétiques, vol. 1, Lausanne, L'Age d'Homme, 1981.

—, Eugène Onéguine, tr. par A. Markowicz, Arles, Actes Sud, 2005.

PušKin, A., Stihotvorenija, partie II, Sankt-Peterburg, Tipografija Departamenta narodnogo prosveščenija, 1829.

Safran, G., "Love Song Between the Sacred and the Vernacular: Pushkin's "Podrazhaniia " in the Context of Bible Translation", Slavic and East European Journal, 39, 2 (1995), p. 165-183.

ŠAPIR, M. I., "Iz istorii russkogo “ balladnogo stiha” : Perom vladeet kak eldoj», in Russian Linguistics, 17, 1 (1993), p. 57-84.

—, «Simvoličeskaja zaum' Fedora Sologuba: meždu ložju i fantaziej», Voprosy literatury, 3 (2007), p. 211-238.

Second, J., Les Baisers et l'épithalame suivis des odes et des élégies, Paris, Garnier Frères, 1938.

Sologub, F., Stihotvorenija, Leningrad, Sovetskij pisatel', 1975. 
Solov'ev, S., Sobranie stihotvorenij, Moskva, Vodolej Publishers, 2007. Tomaševskij, B., «K. N. Batjuškov», in K. Batjuškov, Stihotvorenija, Leningrad, Sovetskij pisatel', 1948, p. V-LX.

Vinogradov, V. V., Jazyk Puškina: Puškin i istorija russkogo literaturnogo jazyka, Moskva/Leningrad, Academia, 1935.

Virgile, Euvres complètes, tr. du latin par Jean-Pierre ChausserieLaprée. Vol. 1, Enéide, Paris, Editions de la Différence, 1993.

Vjazemskij, P. A., "Pis'ma k K. N. Batjuškovu». Publikacija V. A. Košeleva, in Literaturnyj arhiv: Materialy po istorii russkoj literaturu i obščestvennoj mysli, Sankt-Peterburg, Nauka, 1994, p. 118-143. 
